\title{
OPEN Performance controlled via surface oxygen-vacancy in Ti-based oxide catalyst during methyl oleate epoxidation
}

Supareak Praserthdam ${ }^{1,2}$, Meena Rittiruam ${ }^{1,2}$, Kanokpon Maungthong ${ }^{2}$, Tinnakorn Saelee ${ }^{1,2}$ Siriwimol Somdee ${ }^{1,2}$ \& Piyasan Praserthdam ${ }^{2}$

The catalytic performance with high conversion and high selectivity of Ti-based oxide catalysts have been widely investigated. Besides, stability, which is an essential parameter in the industrial process, lacked fundamental understanding. In this work, we combined computational and experimental techniques to provide insight into the deactivation of P25 and TS-1 Ti-based oxide catalysts during the methyl oleate (MO) epoxidation. The considered deactivation mechanisms are fouling and surface oxygen vacancy $\left(\mathrm{O}_{\mathrm{v}}\right)$. The fouling causes temporary catalyst deactivation through active site blockage but can be removed via calcination in air at high temperature. However, in this work, the $\mathrm{O}_{v}$ formation plays an important role in the overall performance of the spent catalyst as the deactivated catalyst after regeneration, cannot be restored to the initial activity. Also, the effects of $O_{v}$ in spent catalysts caused (i) the formation of more $\mathrm{Ti}^{3+}$ species on the surface as evident by XPS and Bader charge analysis, (ii) the activity modification of the active region on the catalyst surface as the reduction in energy gap $\left(E_{g}\right)$ occurred from the formation of the interstates observed in the density of states profiles of spent catalyst modeled by the O-vacant P25 and TS-1 models. This reduction in $\mathrm{E}_{\mathrm{g}}$ affects directly the strength of $\mathrm{Ti}-\mathrm{OOH}$ active site and $\mathrm{MO}$ bonding, in which high binding energy contributes to a low conversion because the $\mathrm{MO}$ needed an $\mathrm{O}$ atom from Ti-OOH site to form the methyl-9,10epoxy stearate. Hence, the deactivation of the Ti-based oxide catalysts is caused not only by the insoluble by-products blocking the active region but also mainly from the $\mathrm{O}_{\mathrm{v}}$. Note that the design of reactive and stable Ti-based oxide catalysts for $\mathrm{MO}$ epoxidation needed strategies to prevent $\mathrm{O}_{\mathrm{V}}$ formation that permanently deactivated the active region. Thus, the interrelation and magnitude between fouling and $\mathrm{O}_{\mathrm{v}}$ formation on catalyst deactivation will be investigated in future works.

Since chemical industries are shifting towards greener technologies, utilizing renewable feedstocks, e.g., biomass, overcomes the shortage of natural resources while reducing environmental is impacted by moving away from petroleum-based supply. In the plant-based oil-producing regions, especially in Southeast Asia, the palm oil is oversupplied. Thus, the conversion of palm oil to other higher-value products is of great interest ${ }^{1}$. One of the targeted products is green diesel, called the fatty acid methyl esters (FAME). It was produced either via the esterification reaction of animal or plant-based oil. ${ }^{2,3}$ However, various energy policies in different countries that encourage the use of electric vehicles to lower the demand for biodiesel suppressed the demand for FAME. Hence, the epoxidation reaction can be the solution towards the utilization of the FAME producing higher-value chemicals, especially, epoxide products ${ }^{4}$ which are used in as plasticizers ${ }^{5}$, stabilizer in PVC, intermediates in polyurethane polyols ${ }^{6}$, lubricants ${ }^{7}$, cosmetics, precursors of various polymer ${ }^{8}$, wood impregnation, biofuel additives ${ }^{9}$, and in pharmaceuticals ${ }^{10}$. So far, the low-temperature liquid-phase epoxidation reaction was described by Prileshajew, as shown in Supplementary Figures S1. The homogeneous reaction is catalyzed by percarboxylic acids which are formed in situ from the reaction between hydrogen peroxide and the carboxylic acid on soluble mineral acids such as $\mathrm{H}_{3} \mathrm{PO}_{4}, \mathrm{HCl}$, or $\mathrm{H}_{2} \mathrm{SO}_{4}^{9,11}$. In this work, an unsaturated FAME, methyl oleate (MO) is studied, where the targeted epoxide product is the methyl-9,10-epoxy stearate (ME). However, a homogeneous process that is used

${ }^{1}$ High-Performance Computing Unit (CECC-HCU), Center of Excellence on Catalysis and Catalytic Reaction Engineering (CECC), Chulalongkorn University, Bangkok 10330, Thailand. ${ }^{2}$ Center of Excellence on Catalysis and Catalytic Reaction Engineering (CECC), Chulalongkorn University, Bangkok 10330, Thailand. ${ }^{\varpi}$ email: piyasan.p@ chula.ac.th 


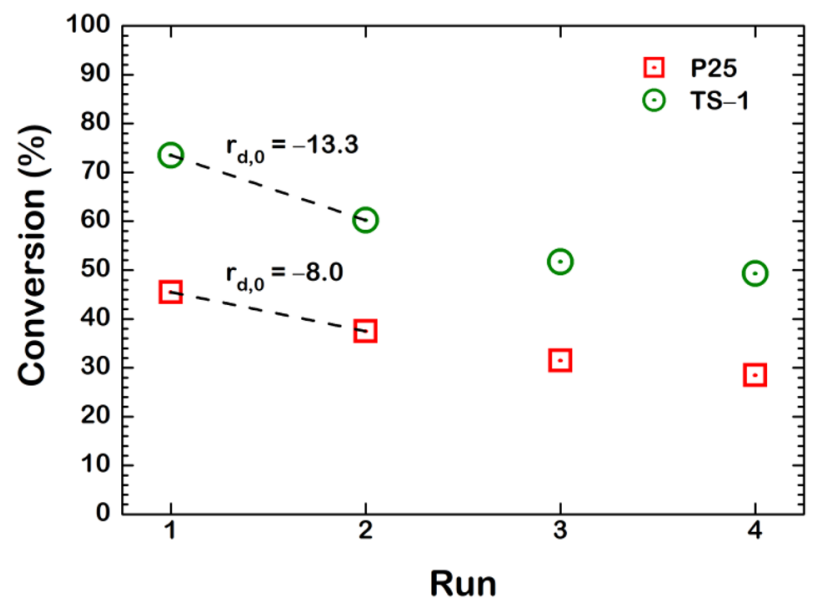

Figure 1. The methyl oleate conversion on P25 (red square) and TS-1 (green circle) catalysts during methyl oleate epoxidation reaction operated in a batch reactor.

\begin{tabular}{|l|l|l|l|}
\hline \multirow{2}{*}{ Catalyst } & \multicolumn{2}{|l|}{ MO conversion (\%) } & \\
\cline { 2 - 4 } & 1st run & 2nd run without calcination & 2nd run calcined at $\mathbf{5 5 0}{ }^{\circ} \mathbf{C}$ \\
\hline P25 & 45.5 & 37.5 & 42.4 \\
\hline TS-1 & 73.5 & 60.2 & 67.2 \\
\hline
\end{tabular}

Table 1. The MO conversion of 1 st run, 2 nd without calcination, and 2 nd run with calcination, for P25 and TS-1.

as catalysts in an industrial process causes corrosion from $\mathrm{H}_{3} \mathrm{PO}_{4}, \mathrm{HCl}$, and $\mathrm{H}_{2} \mathrm{SO}_{4}{ }^{12}$, exhibiting low selectivity since the soluble mineral acid promotes side reactions, e.g., oxirane ring-opening ${ }^{1}$. In addition, the catalyst reuse from a homogeneous process is impractical due to the difficult separation between the catalysts and products. Due to this, the Ti-based oxide catalysts are of interest for the epoxidation reaction. This commercial catalyst is available as titanium dioxide $\left(\mathrm{TiO}_{2}\right)$ with a great number of the Ti-O-Ti moieties known as $\mathrm{P} 25{ }^{1}$. In addition, the ordered structure titanosilicate, TS- 1 containing $\mathrm{SiO}_{2}$-doped $\mathrm{TiO}_{2}$ exhibiting high conversion and selectivity is considered a good candidate ${ }^{12}$ because $\mathrm{SiO}_{2}$ help promote the dispersion of the $\mathrm{TiO}_{2}{ }^{13}$. The active center in FAME epoxidation reaction on the $\mathrm{TiO}_{2}$ is the titanium hydroperoxo species ( $\left.\mathrm{Ti}-\mathrm{OOH}\right)$ formed via the reaction between the added hydrogen peroxide and the clean $\mathrm{TiO}_{2}$ surface. The Ti-OOH active sites converted $\mathrm{MO}$ to ME product and $\mathrm{H}_{2} \mathrm{O}$ as by-product illustrated in Figures $\mathrm{S} 2$ of the supplementary document. To achieve the highest performance for such catalysts, one must tackle its surface deactivation by understanding the underlying mechanism during the liquid-phase epoxidation reaction occurred at low temperature ${ }^{2}$.

Therefore, we investigate the deactivation scheme on P25 and TS-1 Ti-based oxide catalysts during the liquid-phase MO epoxidation reaction at low temperature based on the evidence from experimental data, surface characterizations, and computational data obtained via the density functional theory-based (DFT) analysis ${ }^{14-19}$. Ultimately, the information of such deactivation schemes would be used to construct the guideline towards the design of reactive and stable Ti-based oxide catalysts for epoxidation reactions.

\section{Results and discussion}

To understand the deactivation of the P25 and TS- 1 catalysts, we first determine their stability in terms of MO conversion during the epoxidation reaction on the fresh and used catalysts, as shown in Fig. 1. For the first batch, exactly, the TS- 1 yielded higher conversion at $73.5 \%$ than that of the P25 at $45.5 \%$. The deactivation is then preevaluated via the initial deactivation rate $\left(\mathrm{r}_{\mathrm{d}, 0}\right)$ calculated from the change of $\mathrm{MO}$ conversion over the first and second batch, where the $\mathrm{r}_{\mathrm{d}, 0}$ is -13.3 for the TS- 1 and -8.0 for the P25. Up to this point, the assumption towards the deactivation was proposed to be formed the fouling of either products or by-products, in which the carbon contents may occur in the used catalysts. This concern can be eliminated by the calcination method. As a result, the spent TS- 1 and P25 catalysts from the 1st batch were calcined in air at $550{ }^{\circ} \mathrm{C}$ for $5 \mathrm{~h}$ to confirm the removal of the fouling species. This selected temperature is confirmed to avoid the phase change of our Ti-based oxide catalyst $^{20}$. Thus, the regenerated catalysts should perform with comparable MO conversion to the fresh catalyst if the fouling is the main cause of deactivation.

The MO conversion of fresh catalysts was compared with that of with and without calcinated catalysts as shown in Table 1. It is suggested the removal of the fouling species via calcination can be observed from the increased MO conversion of calcined catalysts. In fact, we found that the MO conversion of the calcined ones was not regenerated back to the value of the fresh catalysts. This raised the concern about our assumption that fouling may not be only the main contributor to the deactivation mechanism. However, the catalyst surface 


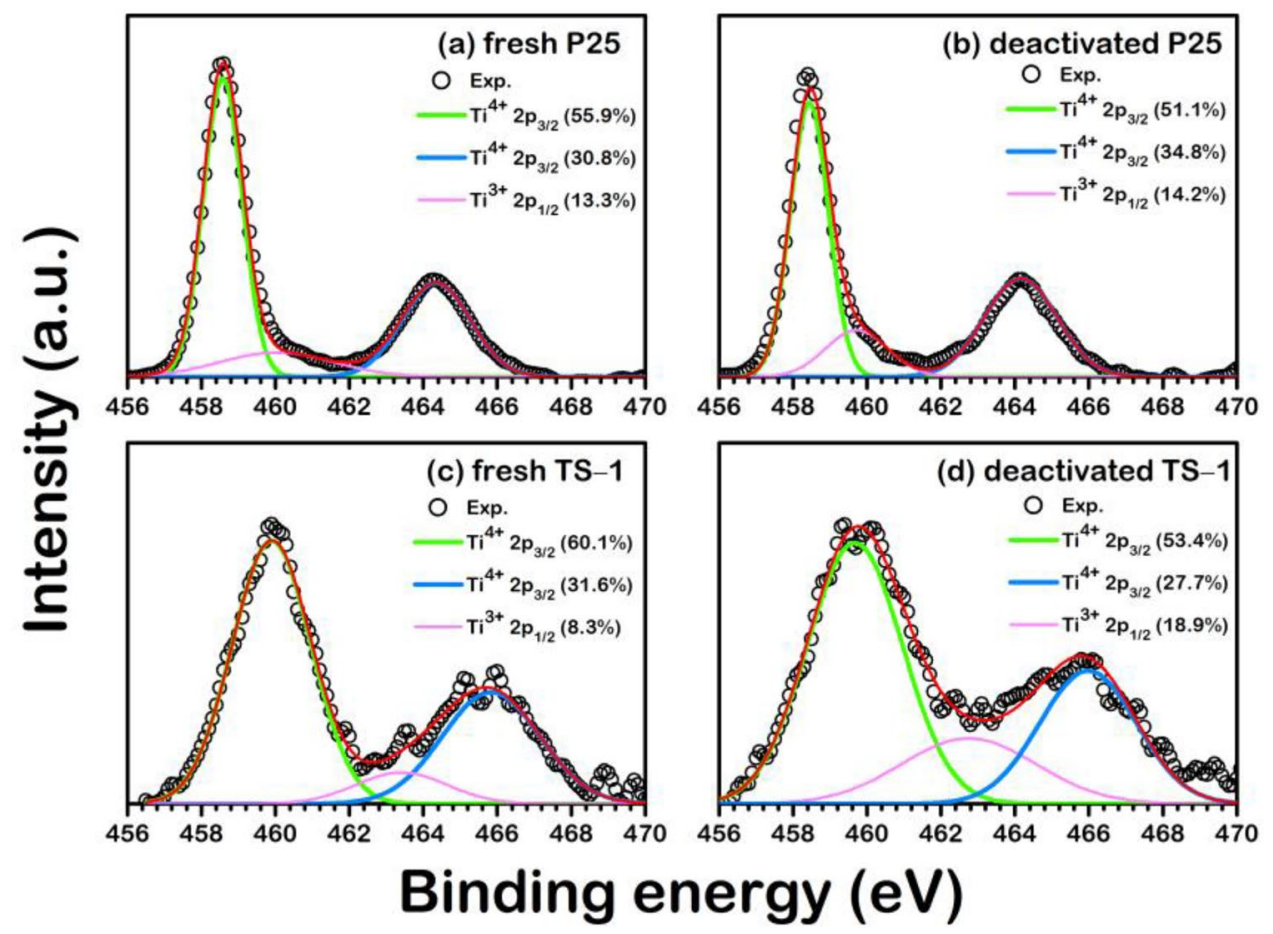

Figure 2. The high-resolution XPS spectra of Ti2p in (a) fresh P25, (b) deactivated P25, (c) fresh TS-1, and (d) deactivated TS-1.

transformation may play a role in the modification of active regions. We intended to investigate the changes in the number of surface species of the elements on the surface: Ti and $\mathrm{O}$ on both catalysts. Hence, the tracking of the surface transformation during the reaction was carried out via the XPS technique.

The high-resolution XPS spectra profiles of P25 and TS-1 illustrated in Figs. 2 and 3 reveal the changes in the amount of $\mathrm{Ti}^{3+}, \mathrm{Ti}^{4+}$, and $\mathrm{O}$ vacancy on the catalyst surfaces. The analyzed XPS is implemented in the Supplementary document as Table S1. On the Ti species of the fresh catalysts, the $\mathrm{Ti}^{4+} 2 \mathrm{p}_{3 / 2}$ peaks were detected at $458.6 \mathrm{eV}$ and $464.2 \mathrm{eV}$ for P25, and $459.9 \mathrm{eV}$ and $465.7 \mathrm{eV}$ for TS-1. These peaks are in good agreement with the anatase $\mathrm{TiO}_{2}{ }^{21}$ and TS- ${ }^{22}$. In addition, the $\mathrm{Ti}^{3+} 2 \mathrm{p}_{1 / 2}$ peak, which was classified to the $\mathrm{Ti}_{2} \mathrm{O}_{3}$ phase ${ }^{23}$, occurred between the peaks of $\mathrm{Ti}^{4+} 2 \mathrm{p}_{3 / 2}$ at $460.4 \mathrm{eV}$ for P25 and $463.4 \mathrm{eV}$ for TS- 1 catalysts. To track the evolution of the catalyst surface during MO epoxidation, the XPS profile in Fig. 2 was analyzed for the changes in the content of surface $\mathrm{Ti}^{3+}$ and $\mathrm{Ti}^{4+}$ species. From Fig. 2a, the fresh TS-1 possessed $8.3 \%$ of $\mathrm{Ti}^{3+}$ species, which is less than that of the P25 (13.3\%), shown in Fig. 2c. On the profiles of spent catalysts shown in Fig. 2b,d, the $\mathrm{Ti}^{3+}$ species increased to $18.9 \%$ and $14.2 \%$ for TS- 1 and P25, respectively. Besides, the changes in surface oxygen vacancies were investigated via the XPS, as shown in Fig. 3. The XPS profile indicates that there are three oxygen species: lattice oxygen $\left(\mathrm{O}_{\mathrm{L}}\right)$, sub oxide $\left(\mathrm{O}_{\mathrm{S}}\right)$, and oxygen vacancy $\left(\mathrm{O}_{\mathrm{V}}\right)$ that are also confirmed by Bharti et al. ${ }^{23}$ It was found in all fresh catalysts that only the $\mathrm{O}_{\mathrm{L}}$ and $\mathrm{O}_{S}$ species were observed, while the $\mathrm{O}_{V}$ was found in all reused catalysts. The $\mathrm{O}_{V}$ observed in the deactivated ones is confirmed that the deactivation during MO epoxidation involves the removal of a surface oxygen atom. Therefore, we hypothesized the formation of these $\mathrm{O}_{\mathrm{V}}$ modified the active region on the catalyst surface, resulting in the reduced activity which leads to reduce the MO conversion. To further clarify such argument on the deactivation mechanism, the DFT analysis was employed in the following section.

To investigate the role of $\mathrm{O}_{\mathrm{V}}$ species formed on the surface of reused catalysts, we first modeled the P25 and TS- 1 surface. From the XRD profile, shown in Supplementary Figures S3, the P25 exhibits anatase as the main phases, while rutile also hardly occurs. The TS-1 shows the ZSM-5-like the structure of high crystalline TS-1, in which the Ti peaks also appear in the TS-1 XRD profile. Therefore, the models in this study were designed based on the XRD profiles.

There are two different methods to design the P25 and TS- 1 catalyst surfaces because of a different crystal structure. For the P25 surface, it is confirmed from the experiment that anatase $\mathrm{TiO}_{2}$ is the main phase. We first calculated the full geometry-optimization in the bulk anatase $\mathrm{TiO}_{2}$, in which the structural parameters are uncovered in Supplementary.

The optimized model of $\mathrm{P} 25$ is illustrated in Fig. 4a. The anatase $\mathrm{TiO}_{2}$ phase was represented by the $\mathrm{TiO}_{2}(101)$ slab model referring to the model construction of Lazzeri et al. ${ }^{24}$ who revealed such $\mathrm{TiO}_{2}(101)$ surface to be the most stable surface of anatase. The surface model comprised of 48 atoms having the formula of $\mathrm{Ti}_{16} \mathrm{O}_{32}$ as $2 \times 2 \times 1$ supercell (Fig. 4 b), where the vacuum is set to $15 \AA$ to avoid the interactions due to the periodicity (Fig. 4c). This surface model shows two Ti-O layers represented the unit cell of anatase $\mathrm{TiO}_{2}(101)$, where the increase of the $\mathrm{Ti}-\mathrm{O}$ layer of this pattern not effects to density of states and still has the same energy gap. In the 

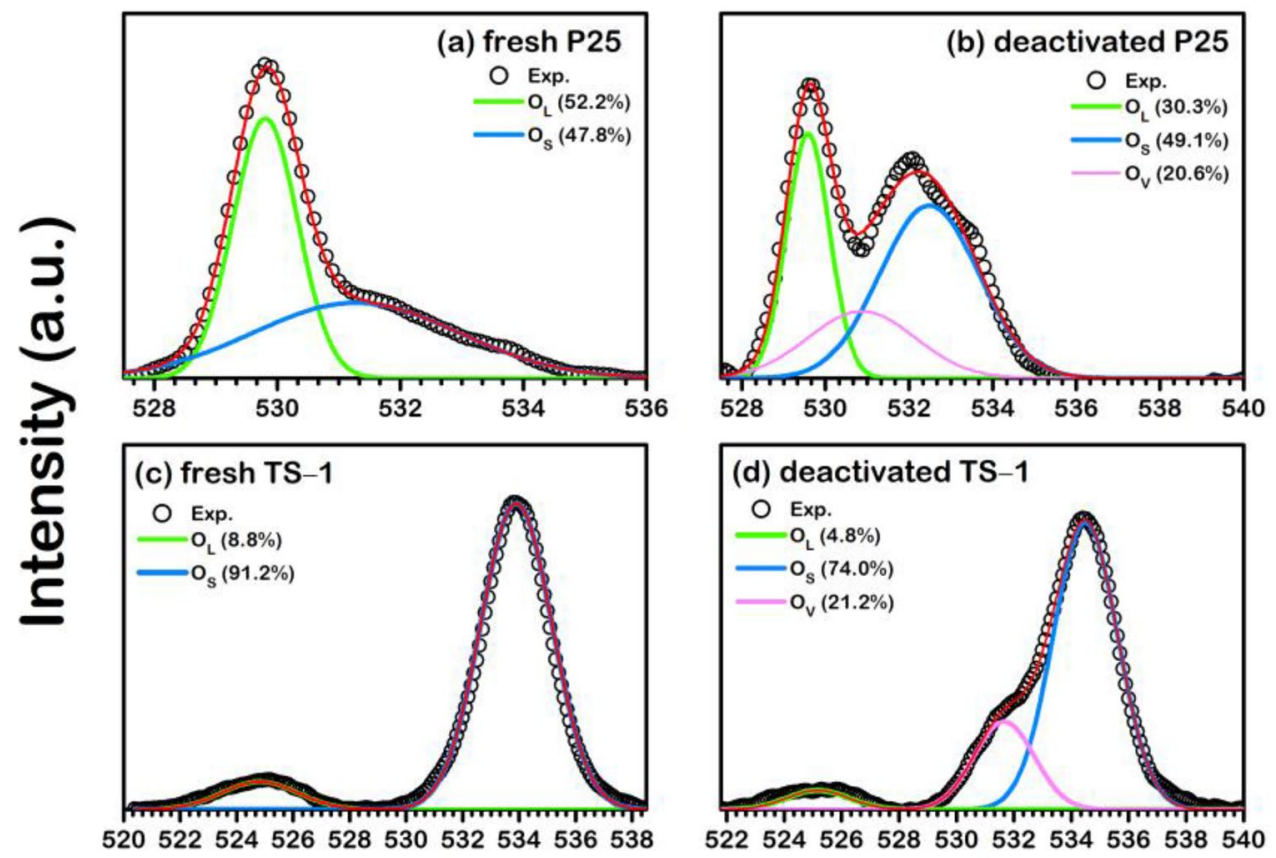

\section{Binding energy (eV)}

Figure 3. The high-resolution XPS spectra of O1s in (a) fresh P25, (b) deactivated P25, (c) fresh TS-1, and (d) deactivated TS-1.
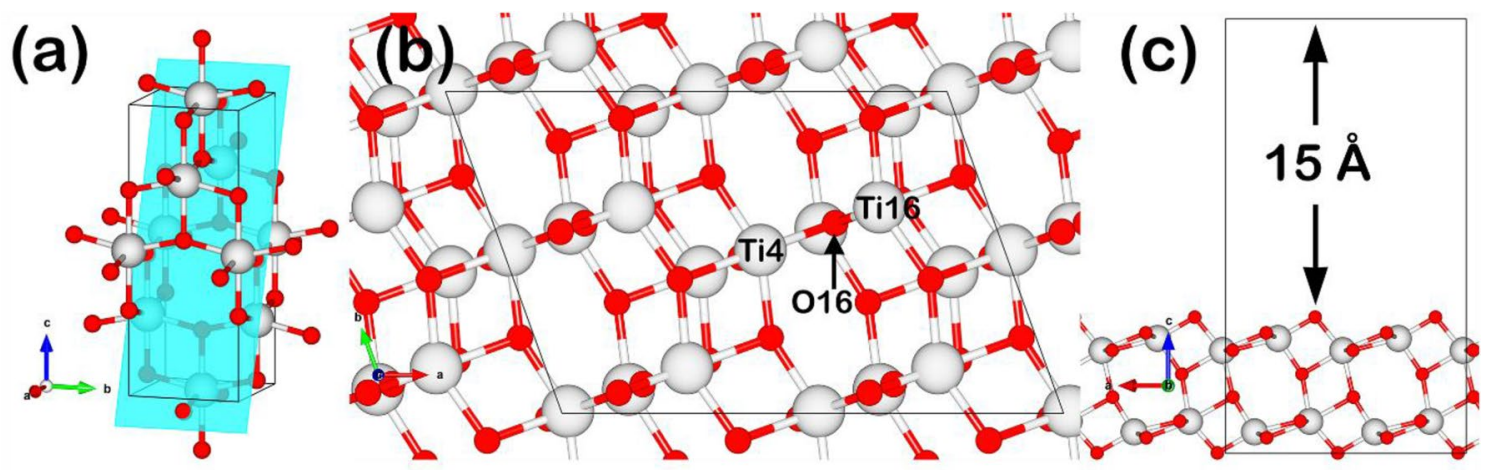

(d)
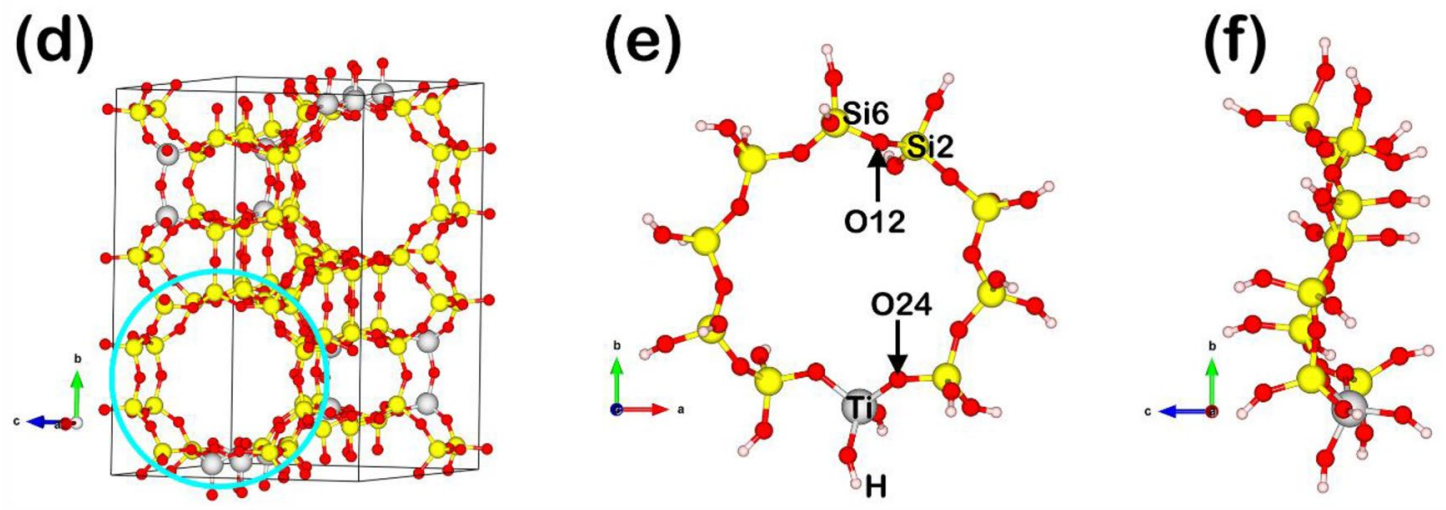

Figure 4. The $\mathrm{P} 25$ and TS- 1 catalyst model. The $\mathrm{P} 25$ is represented by anatase $\mathrm{TiO}_{2}$, showing (a) bulk structure, and $(\mathbf{b}, \mathbf{c})$ top and side views of anatase $\mathrm{TiO}_{2}(101)$. The TS-1 is optimized through the TS-1 (ZSM-5, Ti-doped $\mathrm{SiO}_{2}$ ), including (d) bulk structure, and (b, d) front and side views of TS-1. The designed structure is described in the result and discussion section. 


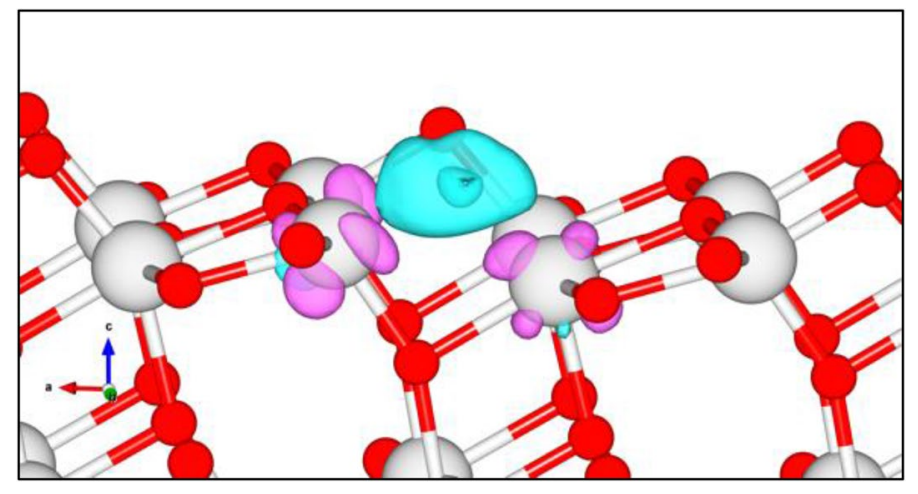

Figure 5. Electron transfer in terms of charge accumulation/depletion regions in the $\mathrm{P} 25$ surface with $\mathrm{O}$ vacancy. The blue regions represent charge accumulation, while the purple regions represent charge depletion.

full geometry-optimization, we use the selective dynamics in order to allow the Ti-O top layer to free relax, while, the Ti-O bottom layer is fixed.

For the TS-1 model, it was designed by the Ti-doped ZSM-5-like structure based on the reported procedures in our previous works ${ }^{25,26}$. In each $10 \mathrm{~T}$ ring, one Ti atom was substituted on one Si atom forming the TS-1 with Ti:Si ratio of 1:5 as illustrated in Fig. 4d. To represent well the real TS- 1 structure, we performed the geometry optimization of the $\mathrm{Si}_{80} \mathrm{Ti}_{16} \mathrm{O}_{192}$ bulk structure shown in Fig. $4 \mathrm{~d}$. Because the reaction occurs in the $10 \mathrm{~T}$ ring rather than in other regions, the TS-1 surface model is also different from the P25. We thus scaled-down the TS- 1 cluster represented by a 9.5 - $\AA$-diameter $10 \mathrm{~T}$ ring to be the reactive region participating in the reaction modeled in a $25 \times 25 \times 15 \AA^{3}$ cubic system.

In addition, to represent the rest of the TS-1 framework, after the optimization of the bulk structure, one hydrogen atom was added to all terminal $\mathrm{O}$ atoms of the $10 \mathrm{~T}$ ring. All dangling $\mathrm{OH}$ groups were fixed to the lattice parameter of the bulk to represent the TS-1 structure before being further optimized. In the geometry optimization, we also used the selective dynamics method to allow atoms to relax except the $\mathrm{OH}$ groups fully. The optimized TS-1 surface model is shown in Fig. 4e,f.

On the representation of the deactivated surface caused by $\mathrm{O}_{\mathrm{V}}$ formation, the $\mathrm{O}_{\mathrm{V}}$ sites on the P25 and TS-1 surfaces were created and modeled by removing an $\mathrm{O}$ atom in each model at the following locations: (1) $\mathrm{O} 16$ in TS-1, (2) $\mathrm{O} 24$ in $\mathrm{P} 25$ representing the $\mathrm{O}_{\mathrm{V}}$ near a Ti-site $\left(\mathrm{O}_{\mathrm{V}, \mathrm{Ti}}\right)$, and (3) O12 in P25 representing the $\mathrm{O}_{\mathrm{V}}$ near a Si-site $\left(\mathrm{O}_{\mathrm{V}, \mathrm{Si}}\right)$, in which the atomic position label is shown in Fig. $4 \mathrm{~b}$ for P25 and Fig. $4 \mathrm{e}$ for TS-1.

Having all the modeled catalysts, the analysis of their electronic properties was carried out by the Bader charge analysis. On the P25 surface, it was found that the formation of $\mathrm{O}_{\mathrm{V}}$ induced charge accumulation in the region between the two Ti atoms ( $\mathrm{Ti} 4$ and $\mathrm{Ti16}$ ) previously connected to the removed $\mathrm{O}$ atom, while the charge of both $\mathrm{Ti}$ atoms depleted as shown in Fig. 5. In addition, from the charge transfers of Ti4 and Ti16, labeled in the Fig. $4 \mathrm{~b}$, decreased from $+2.41|e|$ and $+2.36|e|$ to $+2.03|e|$ and $+1.99|e|$, respectively. This decrease in charge transfer correlated with the decrease in an oxidation state of $\mathrm{Ti}_{\text {f }}$ from $\mathrm{Ti}^{4+}$ to $\mathrm{Ti}^{3+}$ verified by XPS. Hence, the model of P25 can represent well the P25 used in the experiment.

For the TS-1 catalyst, two models of the deactivated surface were constructed: (1) TS-1 with $\mathrm{O}_{V}$ adjacent to the Ti-site (labeled as $\mathrm{O}_{\mathrm{V}, \mathrm{Ti}}$ ) and (2) TS-1 with $\mathrm{O}_{\mathrm{V}}$ far from the Ti-site (labeled as $\mathrm{O}_{\mathrm{V}, \mathrm{Si}}$ ) illustrated in Fig. 6 . The analysis of charge transfer in Table 2 suggested that only the location of $\mathrm{O}_{\mathrm{V}}$ near the Ti-site affects the charge transfer of the Ti atom, whereas the $\mathrm{O}_{\mathrm{V}}$ located far from the Ti-site affected the charge transfer of Si atoms near such $\mathrm{O}_{\mathrm{V}}$ but not the Ti-site.

We further investigated the electronic effects of $\mathrm{O}_{\mathrm{V}}$ on the surface apart from the charge transfer via the analysis of the projected density of state (PDOS). The spin-polarized PDOS of the fresh and deactivated surface of P25 and TS- 1 was obtained and analyzed, as shown in Fig. 7, in which the Fermi level $\left(\mathrm{E}_{\mathrm{F}}\right)$ was set to $0 \mathrm{eV}$; thus, the negative and positive energy represents the valence state and conduction state, respectively. For the P25 catalyst, the energy gap $\left(\mathrm{E}_{\mathrm{g}}\right)$ incorporating the Hubbard $\mathrm{U}$ correction and $\mathrm{D} 3$ dispersion terms is $3.16 \mathrm{eV}(\mathrm{DFT}+U+\mathrm{D} 3$, $U=6.00 \mathrm{eV}$ ), while Portillo-Vélez et al. ${ }^{27}$ previously verified the $\mathrm{E}_{\mathrm{g}}$ to be $2.98 \mathrm{eV}$ with the Hubbard $\mathrm{U}$ correction but without the $\mathrm{D} 3$ dispersion term $(\mathrm{DFT}+U, U=6.00 \mathrm{eV})$. As for this, we suggested that one should include the D3 term to represent well the P25; hence, our calculated band gap is reliable. On the PDOS profile of P25, the O-PDOS (red line) is the major contributor to the total density of state (TDOS) in the valence state, while the Ti-PDOS (blue line) is the main contribution in the TDOS of the conduction state. Analyzing the changes in PDOS of the P25 catalyst after the reaction via the comparison of the PDOS of fresh P25 and deactivated $\mathrm{P} 25$ is revealed that the $\mathrm{E}_{\mathrm{g}}$ of the fresh surface of $3.16 \mathrm{eV}$ reduced to $2.21 \mathrm{eV}$ when the $\mathrm{O}_{\mathrm{V}}$ formed. Also, on the deactivated surface, the conduction band minimum (CBM) shifted from $3.16 \mathrm{eV}$ to $2.17 \mathrm{eV}$, while the valence band maximum (VBM) shifted from $-0.00089 \mathrm{eV}$ to $-1.07 \mathrm{eV}$. Interestingly, with surface $\mathrm{O}_{\mathrm{V}}$, unpaired electronic states were detected between $-0.1 \mathrm{eV}$ and $-0.8 \mathrm{eV}$, comprised mainly of the Ti-state. These peaks correlated with the charge accumulation region confirmed via the Bader charge analysis, illustrated in Fig. 6.

For the TS-1, the calculated $\mathrm{E}_{\mathrm{g}}$ is $4.45 \mathrm{eV}(\mathrm{DFT}+U+\mathrm{D} 3, U=2.00 \mathrm{eV})$. The Hubbard $U$ correction parameter for $\mathrm{Ti}$ in P25 is recommended to be $6 \mathrm{eV}^{27}$, while for other oxide materials, in this case, TS- 1 , the $U$ of $2.00 \mathrm{eV}$ is suggested ${ }^{28}$. Like in the case of P25, the Ti-PDOS is the major contributor in the conduction state, while O-PDOS 


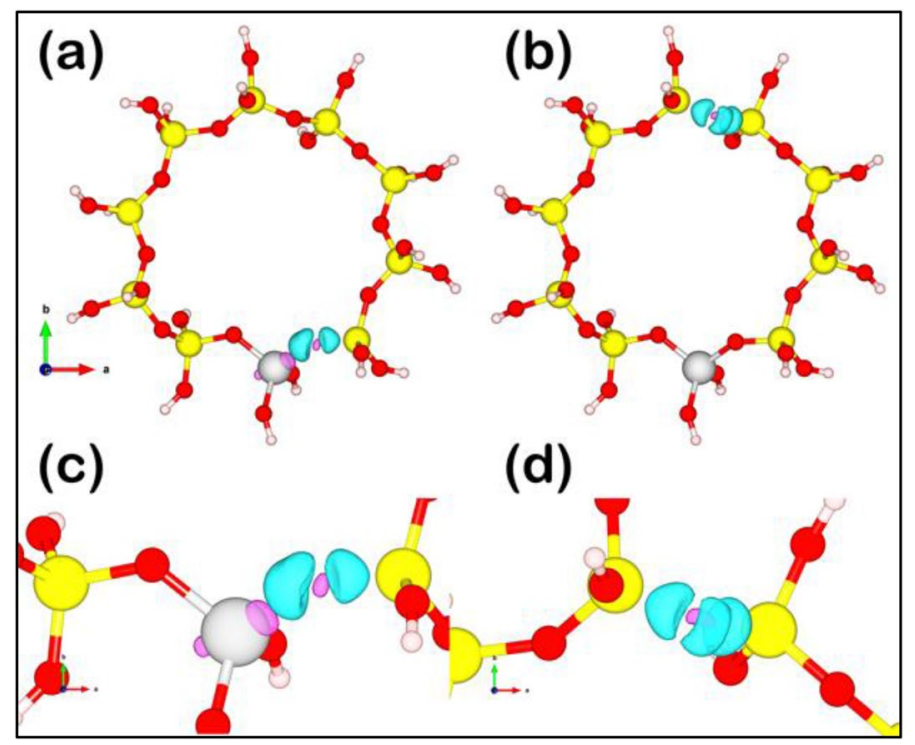

Figure 6. Electron transfer in terms of charge accumulation/depletion regions in TS-1 surface with (a, c) oxygen vacancy near the Ti-site $\left(\mathrm{O}_{\mathrm{V}, \mathrm{Ti}}\right)$ and $(\mathbf{b}, \mathbf{d})$ oxygen vacancy far from the Ti-site $\left(\mathrm{O}_{\mathrm{V}, \mathrm{Si}}\right)$. The blue regions represent charge accumulation, while the purple regions represent charge depletion.

\begin{tabular}{|l|l|l|l|l|l|l|}
\hline Surface type & Ti in top layer & O in top layer & Ti4 & \multicolumn{1}{l|}{ Ti16 } \\
\hline P25 \\
\hline Fresh & +19.10 & -14.28 & +2.41 & +2.36 & \\
\hline With $\mathrm{O}_{\mathrm{V}}$ & +18.28 & -13.35 & +2.03 & +1.99 & \\
\hline Surface type & All Si & All O & Ti & Si2 & Si6 \\
\hline TS-1 & ( \\
\hline Fresh & +28.66 & -44.94 & +2.28 & +3.17 & +3.19 \\
\hline With $\mathrm{O}_{\mathrm{V}, \mathrm{Ti}}$ & +27.03 & -43.29 & +2.07 & +3.18 & +3.19 \\
\hline With $\mathrm{O}_{\mathrm{V}, \mathrm{Si}}$ & +27.47 & -43.49 & +2.28 & +2.38 & +2.40 \\
\hline
\end{tabular}

Table 2. The calculated charge transfer of P25 and TS-1. ${ }^{\star}$ All labeled atoms correlated to the label in Fig. 4.

is the major contributor in the valence state. The $\mathrm{E}_{\mathrm{g}}$ value of the fresh surface at $4.45 \mathrm{eV}$ reduced to $4.02 \mathrm{eV}$ and $2.42 \mathrm{eV}$ for the surface with $\mathrm{O}_{\mathrm{V}, \mathrm{Si}}$ and $\mathrm{O}_{\mathrm{V}, \mathrm{Ti}}$, respectively. The changes of CBM and VBM in the deactivated TS-1 are also similar to that of P25. The CBM shifted from $4.41 \mathrm{eV}$ to $3.92 \mathrm{eV}$ in the case of $\mathrm{O}_{\mathrm{V}, \mathrm{Si}}$ and shifted to $2.38 \mathrm{eV}$ in $\mathrm{O}_{\mathrm{V}, \mathrm{Ti}}$, while, the VBM shifted from $-0.035 \mathrm{eV}$ to $-0.556 \mathrm{eV}$ in the case of $\mathrm{O}_{\mathrm{V}, \mathrm{Si}}$ and shifted to $-1.19 \mathrm{eV}$ in $\mathrm{O}_{\mathrm{V}, \mathrm{Ti}}$. However, unlike the P25 surface with $\mathrm{O}_{V}$, the unpaired electronic states were found. The paired electronic state was detected in the valence state between $-0.0006 \mathrm{eV}$ and $-0.417 \mathrm{eV}$ for the TS- 1 with $\mathrm{O}_{\mathrm{V}, \mathrm{Si}}$, while the one with $\mathrm{O}_{\mathrm{V}, \mathrm{Ti}}$ such state, is located between $-0.035 \mathrm{eV}$ and $-0.42 \mathrm{eV}$. Both of the paired electronic states comprised the $\mathrm{Si}$, Ti, and $\mathrm{O}$ states equally. Besides, in the conduction state, an interstate was also found at $2.5 \mathrm{eV}$ only for the TS-1 with $\mathrm{O}_{\mathrm{V}, \mathrm{Ti}}$.

As the calculated PDOS, which is indicated that the $\mathrm{O}_{\mathrm{V}}$ induced the formation of the interstate leading to decrease $E_{g}$, the UV-vis profiles of the fresh and spent catalysts are obtained and analyzed, shown in Fig. 8, in order to confirm these phenomena. The UV-vis profiles plotted the absorbance against energy were used to investigate the $\mathrm{E}_{\mathrm{g}}$ for each sample. It is revealed that both spent P25 and TS-1 surface exhibited a lower $\mathrm{E}_{\mathrm{g}}$ than their fresh surface. In addition, the $\mathrm{E}_{\mathrm{g}}$ of TS-1 surface, which has a higher initial rate of deactivation, reduced more than that of the P25 surface.

Referring to the hypothesis for the deactivation mechanism, the evidence from the characterizations of the surface electronic properties of the fresh and spent P25 and TS- 1 revealed the $\mathrm{O}_{\mathrm{V}}$ is one of the causes altering the activity of the active region on the catalyst surface in addition to the fouling which can be handled via the calcination in air. This deactivation via $\mathrm{O}_{\mathrm{V}}$ occurred through not only the changes in charge transfer, which promoted more $\mathrm{Ti}^{3+}$ species on the surface but also through the formation of the interstates found in the PDOS profile that altered the ability of the active region of the catalyst surface. The direct effect causing to lower conversion is the $\mathrm{Ti}^{3+}$ induced high binding energy between the active site, $\mathrm{Ti}-\mathrm{OOH}$, and $\mathrm{MO}$. It can be implied that charge accumulation makes bonding of $\mathrm{Ti}-\mathrm{OOH}$ strong. Therefore, the conversion of $\mathrm{ME}$, which is formed by one $\mathrm{O}$ atom form $\mathrm{Ti}-\mathrm{OOH}$ and $\mathrm{MO}$, is significantly decreased. 

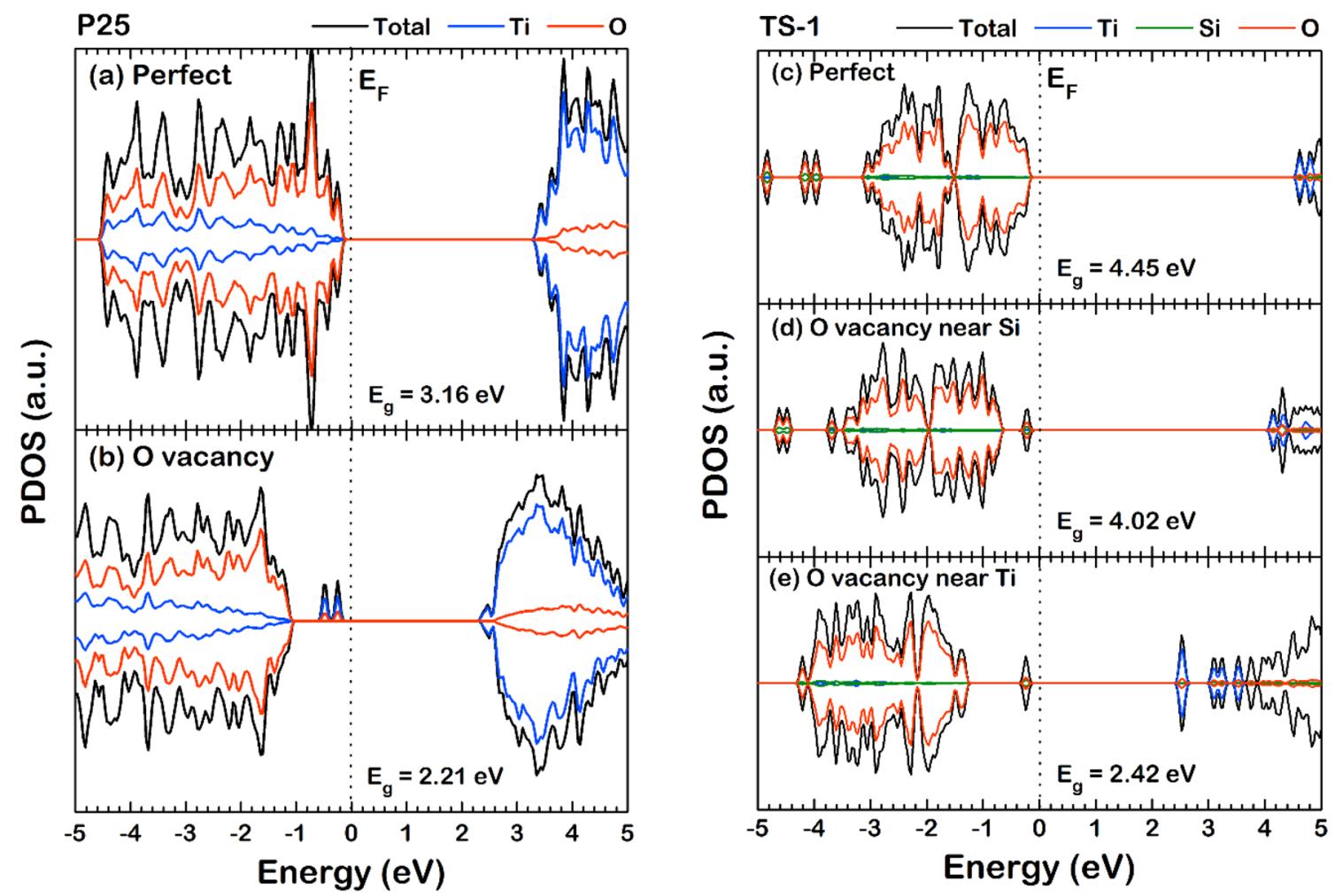

Figure 7. The projected density of state (PDOS) of (a) the fresh and (b) O vacancy for the P25 catalyst surface, and (c) the fresh, (d) the $\mathrm{O}_{\mathrm{V}, \mathrm{Si}}$, and (e) the $\mathrm{O}_{\mathrm{V}, \mathrm{Ti}}$ for TS-1 catalyst surface, respectively.

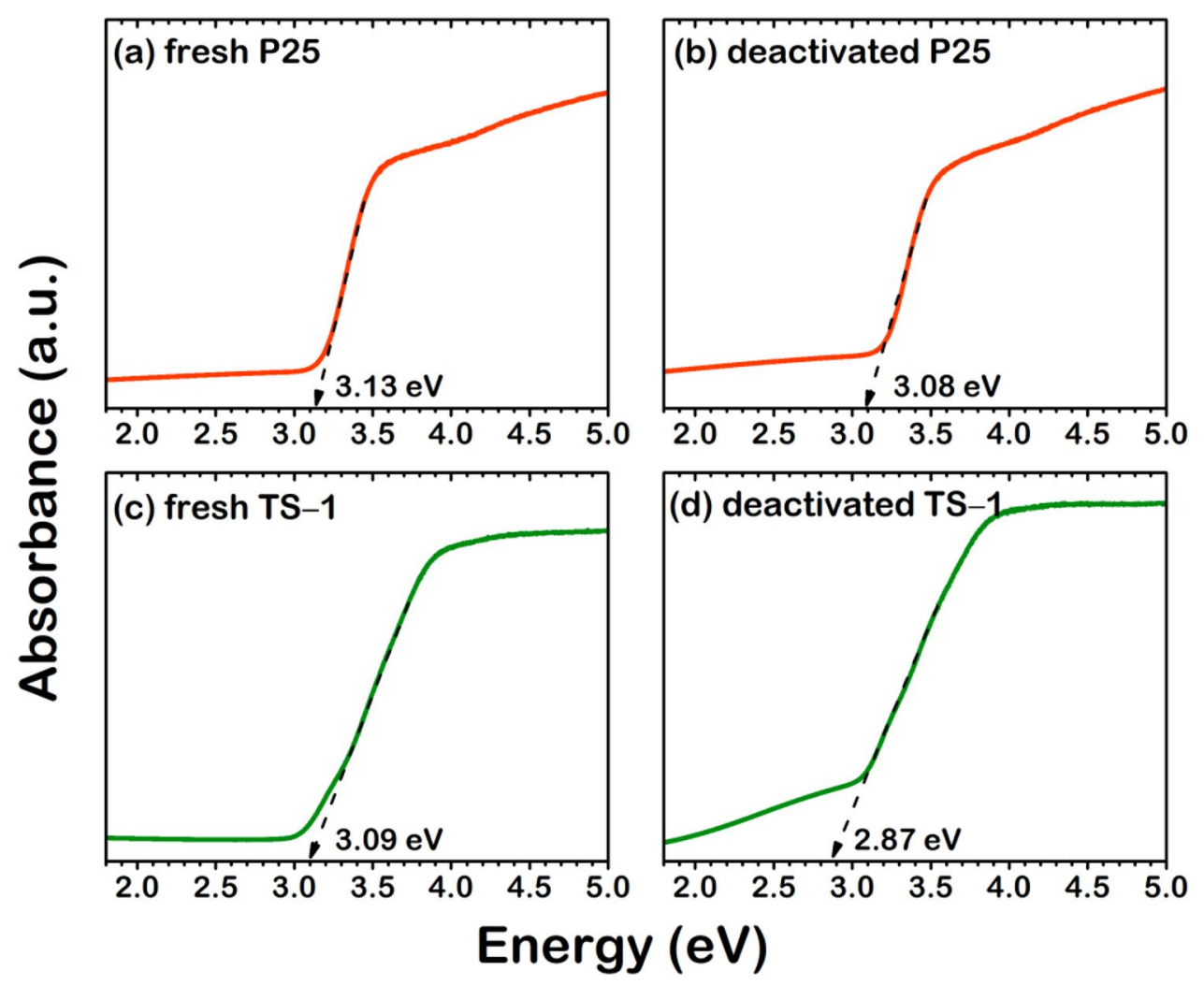

Figure 8. UV-vis spectrometry profile of (a) fresh P25 surface, (b) deactivated P25 surface, (c) fresh TS-1 surface, and (d) deactivated TS-1 surface. 


\section{Conclusion}

The following summarizes the deactivation mechanism of the P25 and TS-1 Ti-based oxide catalysts during the MO epoxidation.

(i) The fouling causes catalyst deactivation but in a regenerative way as the activity of the catalyst can be restored via the calcination in air oxidizing all the fouling species.

(ii) The catalyst surface also deactivated through the loss of oxygen atom, forming the surface $\mathrm{O}_{\mathrm{V}}$ which induced the formation of the interstates in conduction and valence states of the catalyst, where these interstates resulted in the decrease in energy gap for both catalysts leading to the modification of the activity of the active region on the surface.

(iii) The $\mathrm{O}_{\mathrm{V}}$ formation introduces a new electron-rich site on the deactivated surface in both P25, and TS-1 confirmed via the Bader charge analysis, where the charge accumulation is observed in the $\mathrm{O}_{\mathrm{V}}$ site.

Therefore, as the cause of the reduced activity stemmed from the formation of the $\mathrm{O}_{\mathrm{V}}$, the addition of the oxygen atom back to the same removal site would restore the activity during the reaction. However, during the reaction when the $\mathrm{O}$ atom was removed, the surface may have transformed into a more stable surface configuration prior to the addition of the oxygen atom via calcination. This way, the addition of the oxygen atom back to the removed site would not be possible, resulting in the permanent loss of the active region on the catalyst surface.

Ultimately, it was proposed that the cause of deactivation in Ti-based oxide catalysts during MO epoxidation is the combination of fouling and $\mathrm{O}_{\mathrm{V}}$ formation. The fouling causes temporary deactivation and can be cured via calcination that oxidized the fouling species, while the $\mathrm{O}_{\mathrm{V}}$ formation resulted in the surface modification that reduced the activity of the whole catalyst by altering the electronic properties of the active region on the surface producing a number of less active regions.

\section{Methodology}

Experimental details. Catalyst synthesis. The $\mathrm{P} 25$ catalyst was used the commercial $\mathrm{TiO}_{2}$, powder from Aeoxide (formerly Degussa), while the TS-1 catalyst was prepared by the hydrolysis of Ti(IV) isopropoxide (TIP, 97\%, Sigma Aldrich) as Ti precursor and tetraethyl orthosilicate (TEOS, $>99 \%$, Merck) as Si precursor by ammonium hydroxide $\left(\mathrm{NH}_{4} \mathrm{OH}, 28 \%\right.$ solution, Sigma Aldrich). The isopropanol was used as the solvent in order to dissolve TIP in deionized water (DI water) prior to the transfer to autoclave. The synthetic gel formed with a molar ratio of $1.0 \mathrm{TiO}_{2}: 7.4 \mathrm{SiO}_{2}: 6.2\left(\mathrm{NH}_{4}\right)_{2} \mathrm{O}: 1.8 \mathrm{TPABr}: 553.7 \mathrm{H}_{2} \mathrm{O}$ in the $100 \mathrm{~cm}^{3}$ PTFE autoclave was heated at $1{ }^{\circ} \mathrm{C} / \mathrm{min}$ to $160^{\circ} \mathrm{C}$ and held for $2 \mathrm{~h}$ prior to the second heating to $210^{\circ} \mathrm{C}$ at $10^{\circ} \mathrm{C} / \mathrm{min}$ for $5 \mathrm{~h}$. The synthesized crystal was filtered, washed with DI water, and dried in air at $383 \mathrm{~K}$ overnight. The obtained solid was calcined at $550{ }^{\circ} \mathrm{C}$ for $6 \mathrm{~h}$ in air.

Catalyst characterization. The P25 and TS-1 catalysts were characterized by X-ray diffractometer (Bruker $\mathrm{D} 8$ Advance) using $\mathrm{Cu} \mathrm{Ka}$ radiation with $2 \theta$ between $20^{\circ}$ to $80^{\circ}$ for $\mathrm{P} 25$ and $5^{\circ}$ to $50^{\circ}$ for TS- 1 , respectively, with a scan speed of $0.5 \mathrm{~s} / \mathrm{step}$. The elemental contents of $\mathrm{Ti}$ and $\mathrm{O}$ were analyzed by the X-ray photoelectron spectroscopy (XPS), where the spectra were obtained by AMICUS spectrometer using Mg Ka X-ray radiation $(1253.6 \mathrm{eV})$ and $\mathrm{Al} \mathrm{Ka} \mathrm{X-ray} \mathrm{radiation}(1486.6 \mathrm{eV})$ at a voltage of $15 \mathrm{kV}$ and current of $12 \mathrm{~mA}$. The energy gap for each catalyst was derived by the absorbance versus energy profiles obtained by the Ultraviolet-visible spectrophotometry (UV-vis), in which the absorbance from 200 to $500 \mathrm{~nm}$ is obtained by Perkin Elmer Lambda 650 spectrophotometer, where the step size for the scan is $1 \mathrm{~nm}$.

Catalytic reaction testing. The catalytic property of each catalyst was carried out in a $50 \mathrm{~cm}^{3}$ three-necked round-bottom glass reactor with a reflux condenser. During the liquid-phase MO epoxidation reaction at $50{ }^{\circ} \mathrm{C}$, the mixture was stirred with a magnetic stirrer at $500 \mathrm{rpm}$ with a reaction of $5 \mathrm{~h}$. The substrates comprise methyl oleate (MO, '99\%, Sigma-Aldrich) and hydrogen peroxide $\left(\mathrm{H}_{2} \mathrm{O}_{2}, 30\right.$ wt.\% in $\mathrm{H}_{2} \mathrm{O}$, Sigma-Aldrich) as an oxidizing reagent and acetonitrile $\left(\mathrm{CH}_{3} \mathrm{CN}, 99.8 \%\right.$, Sigma-Aldrich) as the solvent. The $\mathrm{MO} / \mathrm{H}_{2} \mathrm{O}_{2}$ feed molar ratio is 3:1, where the naphthalene was used as an internal standard $\left(\mathrm{C}_{10} \mathrm{H}_{8},{ }^{\prime} 98 \%\right.$, Fluka). In addition, the catalyst weighed $0.3 \mathrm{~g}$, was mixed with $\mathrm{MO}$ before added with $\mathrm{H}_{2} \mathrm{O}_{2}$. After each batch of reaction, the catalyst will be collected and reused to analyze the stability over time. The recovering process entailed separation via centrifugation, washed with DI water, and dried overnight at $110^{\circ} \mathrm{C}$. Note that, in the studying of regeneration, the samples were further calcined in air at $550^{\circ} \mathrm{C}$ for $5 \mathrm{~h}$, but apart from that, all reused catalysts were followed the recovery step up to drying only. The product distribution for each run was analyzed by gas chromatography-mass spectrometry (GC-MS) equipped with a DB-5 column with a dimension of $30 \mathrm{~m} \times 0.25 \mathrm{~mm} \times 0.25 \mu \mathrm{m}$ from Shimadzu.

Computational details. The DFT with the projector augmented wave (PAW) implemented in the Vienna ab initio simulation package (VASP) ${ }^{29-32}$ was used to investigate the surface structure and electronic properties. The exchange-correlation functional along with the generalized gradient approximation (GGA) by Perdew, Burke, and Ernzerhof (PBE), especially the GGA $+U$ was used ${ }^{33}$. The parameter $U$ of 6.0 and 2.0 was used for Ti in P25 ${ }^{27}$ and TS- $1{ }^{28}$, respectively. The cut-off energy of $400 \mathrm{eV}$ for P25 and $500 \mathrm{eV}$ for TS-1 were used for the self-consistent loop. The structural optimization (OPT) was performed within the conjugate gradient method ${ }^{34}$ and relaxed until the force convergence less than $0.01 \mathrm{eV} / \AA$. Also, the Van der Waals dispersion term, DFT-D3 proposed by Grimme et al. ${ }^{35}$ was applied for all calculations. In the Monkhorst-Pack $k$-mesh Brillouin-zone integration ${ }^{36}$, the $3 \times 3 \times 1$ and $3 \times 3 \times 3$ were used for P25 and TS- 1 during the OPT. In the density of state (DOS) 
calculation, the value of $5 \times 5 \times 1$ and $5 \times 5 \times 5$ was used for P25 and TS- 1 . All structure models were drawn and visualized by VESTA package ${ }^{37}$.

The partial charge accumulation or depletion during $\mathrm{O}$ vacancy formation $\left(\Delta \delta_{\mathrm{O}-\mathrm{vac}}\right)$ on the surfaces was calculated based on the Bader charge analysis ${ }^{38-41}$ as follows:

$$
\Delta \delta_{\mathrm{O}-\mathrm{vac}}=\delta_{\mathrm{O}-\mathrm{vac}, \text { Sur }}-\delta_{\text {Fresh,Sur }}+\delta_{\mathrm{O}}
$$

The parameters $\delta_{\mathrm{O}-\mathrm{vac}, \text { Sur }}, \delta_{\mathrm{Fresh}, \text { Sur }}$, and $\delta_{\mathrm{O}}$ are the partial charge of the surface with $\mathrm{O}$ vacancy, clean surface, and an $\mathrm{O}$ atom, respectively. Additionally, the methodology of designed models is revealed in the Results and Discussion section.

\section{Data availability}

The authors declare that relevant data are within the manuscript.

Received: 20 July 2020; Accepted: 20 October 2020

Published online: 03 November 2020

\section{References}

1. Knothe, G., Dunn, R. O. \& Bagby, M. O. Biodiesel: The Use of Vegetable Oils and Their Derivatives as Alternative Diesel Fuels (ACS Publications, Washington, DC, 1997).

2. Praserthdam, S. \& Jongsomjit, B. Observation on different turnover number in two-phase acid-catalyzed esterification of dilute acetic acid and 1-heptanol. Catal. Lett. 130(3-4), 583-587 (2009).

3. Praserthdam, S., Wongmaneenil, P. \& Jongsomjit, B. Investigation of different modifiers for nanocrystal zirconia on W/ZrO2 catalysts via esterification. J. Ind. Eng. Chem. 16(6), 935-940 (2010).

4. Camblor, M., Corma, A., Esteve, P., Martinez, A. \& Valencia, S. Epoxidation of unsaturated fatty esters over large-pore Ti-containing molecular sieves as catalysts: important role of the hydrophobic-hydrophilic properties of the molecular sieve. Chem. Commun. 8, 795-796 (1997).

5. Galli, F., Nucci, S., Pirola, C. \& Bianchi, C. Epoxy methyl soyate as bio-plasticizer: two different preparation strategies. Chem. Eng. Trans. 37, 601-606 (2014).

6. Li, Z. et al. Catalytic synthesis of carbonated soybean oil. Catal. Lett. 123(3-4), 246-251 (2008).

7. Borugadda, V. B. \& Goud, V. V. Epoxidation of castor oil fatty acid methyl esters (COFAME) as a lubricant base stock using heterogeneous ion-exchange resin (IR-120) as a catalyst. Energy Procedia 54, 75-84 (2014).

8. Wei, Y., Li, G., Lü, Q., Cheng, C. \& Guo, H. Green and efficient epoxidation of methyl oleate over hierarchical TS-1. Chin. J. Catal. 39(5), 964-972 (2018).

9. Yao, M.-Y., Huang, Y.-B., Niu, X. \& Pan, H. Highly efficient silica-supported peroxycarboxylic acid for the epoxidation of unsaturated fatty acid methyl esters and vegetable oils. ACS Sustain. Chem. Eng. 4(7), 3840-3849 (2016).

10. Grinberg, S., Kipnis, N., Linder, C., Kolot, V. \& Heldman, E. Asymmetric bolaamphiphiles from vernonia oil designed for drug delivery. Eur. J. Lipid Sci. Technol. 112(1), 137-151 (2010).

11. Turco, R., Pischetola, C., Tesser, R., Andini, S. \& Di Serio, M. New findings on soybean and methylester epoxidation with alumina as the catalyst. RSC Adv. 6(38), 31647-31652 (2016).

12. Smeets, V., Mustapha, L. B., Schnee, J., Gaigneaux, E. M. \& Debecker, D. P. Mesoporous SiO2-TiO2 epoxidation catalysts: Tuning surface polarity to improve performance in the presence of water. Mol. Catal. 452, 123-128 (2018).

13. Cozzolino, M., Di Serio, M., Tesser, R. \& Santacesaria, E. Grafting of titanium alkoxides on high-surface SiO2 support: An advanced technique for the preparation of nanostructured TiO2/SiO2 catalysts. Appl. Catal. A 325(2), 256-262 (2007).

14. Rittiruam, M., Yangthaisong, A. \& Seetawan, T. Enhancing the thermoelectric properties of TiNiSn by transition metals co-doped on the Ti-site of Ti0.5TMI0.25TMII0.25NiSn: a first-principles study. J. Appl. Phys. 124(17), 175101 (2018).

15. Rittiruam, M., Yangthaisong, A. \& Seetawan, T. Enhancing the Thermoelectric Performance of Self-Defect TiNiSn: A FirstPrinciples Calculation. J. Electron. Mater. 47(12), 7456-7462 (2018).

16. Rittiruam, M., Jongsomjit, B. \& Praserthdam, S. A computational-experimental investigation on high ethylene selectivity in ethanol dehydration reaction found on WO x/ZrO 2-activated carbon bi-support systems. Sci. Rep. 9(1), 1-9 (2019).

17. Rittiruam, M., Yangthaisong, A. \& Seetawan, T. Reduced lattice thermal conductivity of Ti-site substituted transition metals TilXTMXNiSn: a quasi-harmonic Debye model study. Chin. J. Phys. 57, 393-402 (2019).

18. Saelee, T., Namuangruk, S., Kungwan, N. \& Junkaew, A. Theoretical insight into catalytic propane dehydrogenation on Ni (111). J. Phys. Chem. C 122(26), 14678-14690 (2018).

19. Praserthdam, S., Somdee, S., Rittiruam, M. \& Balbuena, P. B. Computational study of the evolution of Ni-based catalysts during the dry reforming of methane. Energy Fuels 34(4), 4855-4864 (2020).

20. Romeiro, A., Freitas, D., Azenha, M. E., Canle, M. \& Burrows, H. D. Effect of the calcination temperature on the photocatalytic efficiency of acidic sol-gel synthesized $\mathrm{TiO}_{2}$ nanoparticles in the degradation of alprazolam. Photochem. Photobiol. Sci. 16(6), 935-945 (2017).

21. Sanjines, R. et al. Electronic structure of anatase $\mathrm{TiO}_{2}$ oxide. J. Appl. Phys. 75(6), 2945-2951 (1994).

22. Hasegawa, Y. \& Ayame, A. Investigation of oxidation states of titanium in titanium silicalite-1 by X-ray photoelectron spectroscopy. Catal. Today 71(1-2), 177-187 (2001).

23. Bharti, B., Kumar, S., Lee, H.-N. \& Kumar, R. Formation of oxygen vacancies and $\mathrm{Ti}^{3+}$ state in $\mathrm{TiO}_{2}$ thin film and enhanced optical properties by air plasma treatment. Sci. Rep. 6, 32355 (2016).

24. Lazzeri, M., Vittadini, A. \& Selloni, A. Structure and energetics of stoichiometric $\mathrm{TiO}_{2}$ anatase surfaces. Phys. Rev. B 63(15), 155409 (2001).

25. Nahreen, S. et al. Catalytic upgrading of methane to higher hydrocarbon in a nonoxidative chemical conversion. Energy Fuels 30(4), 2584-2593 (2016).

26. Gao, J. et al. Structure of Mo2Cx and Mo4Cx molybdenum carbide nanoparticles and their anchoring sites on ZSM-5 zeolites. J. Phys. Chem. C 118(9), 4670-4679 (2014).

27. Portillo-Vélez, N., Olvera-Neria, O., Hernández-Pérez, I. \& Rubio-Ponce, A. Localized electronic states induced by oxygen vacancies on anatase $\mathrm{TiO}_{2}$ (101) surface. Surf. Sci. 616, 115-119 (2013).

28. Hu, Z. \& Metiu, H. Choice of U for DFT+ U calculations for titanium oxides. J. Phys. Chem. C 115(13), 5841-5845 (2011).

29. Kresse, G. \& Hafner, J. Ab initio molecular dynamics for liquid metals. Phys. Rev. B 47(1), 558 (1993).

30. Kresse, G. \& Hafner, J. Ab initio molecular-dynamics simulation of the liquid-metal-amorphous-semiconductor transition in germanium. Phys. Rev. B 49(20), 14251 (1994).

31. Kresse, G. \& Furthmüller, J. Efficiency of ab-initio total energy calculations for metals and semiconductors using a plane-wave basis set. Comput. Mater. Sci. 6(1), 15-50 (1996). 
32. Kresse, G. \& Furthmüller, J. Efficient iterative schemes for ab initio total-energy calculations using a plane-wave basis set. Phys. Rev. B 54(16), 11169 (1996).

33. Perdew, J. P., Burke, K. \& Ernzerhof, M. Generalized gradient approximation made simple. Phys. Rev. Lett. 77(18), 3865 (1996).

34. Teter, M. P., Payne, M. C. \& Allan, D. C. Solution of Schrödinger's equation for large systems. Phys. Rev. B 40(18), 12255 (1989).

35. Grimme, S., Antony, J., Ehrlich, S. \& Krieg, H. A consistent and accurate ab initio parametrization of density functional dispersion correction (DFT-D) for the 94 elements H-Pu. J. Chem. Phys. 132(15), 154104 (2010).

36. Monkhorst, H. J. \& Pack, J. D. Special points for Brillouin-zone integrations. Phys. Rev. B 13(12), 5188 (1976).

37. Momma, K. \& Izumi, F. VESTA: a three-dimensional visualization system for electronic and structural analysis. J. Appl. Crystallogr. 41(3), 653-658 (2008).

38. Henkelman, G., Arnaldsson, A. \& Jónsson, H. A fast and robust algorithm for Bader decomposition of charge density. Comput. Mater. Sci. 36(3), 354-360 (2006).

39. Tang, W., Sanville, E. \& Henkelman, G. A grid-based Bader analysis algorithm without lattice bias. J. Phys.: Condens. Matter 21(8), 084204 (2009).

40. Sanville, E., Kenny, S. D., Smith, R. \& Henkelman, G. Improved grid-based algorithm for Bader charge allocation. J. Comput. Chem. 28(5), 899-908 (2007).

41. Yu, M. \& Trinkle, D. R. Accurate and efficient algorithm for Bader charge integration. J. Chem. Phys. 134(6), 064111 (2011).

\section{Acknowledgment}

This research is supported by Second Century Fund (C2F), Chulalongkorn University. Also, the experimental results are supported by "Innovation and technology foresight in catalysis and catalytic reaction engineering for biodiesel, ethanol, and catalyst-related industry for sustainable development (CAT-REC Industrial Project)." The Grant for Research Development, Faculty of Engineering, Chulalongkorn University, and the Seed Project from the Department of Chemical Engineering, Faculty of Engineering, Chulalongkorn University, Thailand, are highly acknowledged.

\section{Author contributions}

M.R., T.S., S.S., and S.P. conceived the computational simulations. M.R., T.S., and S.S. performed computational simulations. K.M. and P.P conceived the experiments. K.M. performed the experiments. M.R., T.S., S.S., and S.P. performed computational data analyses. K.M., M.R., T.S., S.P., and P.P. performed experimental data analyses. The paper was written by M.R., K.M., S.P., T.S., and P.P. All authors reviewed the manuscript.

\section{Competing interests}

The authors declare no competing interests.

\section{Additional information}

Supplementary information is available for this paper at https://doi.org/10.1038/s41598-020-76094-2.

Correspondence and requests for materials should be addressed to P.P.

Reprints and permissions information is available at www.nature.com/reprints.

Publisher's note Springer Nature remains neutral with regard to jurisdictional claims in published maps and institutional affiliations.

(c) (i) Open Access This article is licensed under a Creative Commons Attribution 4.0 International License, which permits use, sharing, adaptation, distribution and reproduction in any medium or format, as long as you give appropriate credit to the original author(s) and the source, provide a link to the Creative Commons licence, and indicate if changes were made. The images or other third party material in this article are included in the article's Creative Commons licence, unless indicated otherwise in a credit line to the material. If material is not included in the article's Creative Commons licence and your intended use is not permitted by statutory regulation or exceeds the permitted use, you will need to obtain permission directly from the copyright holder. To view a copy of this licence, visit http://creativecommons.org/licenses/by/4.0/.

(C) The Author(s) 2020 